\title{
Ferroportin mutation in autosomal dominant hemochromatosis: loss of function, gain in understanding
}

\author{
Robert E. Fleming ${ }^{1}$ and William S. Sly ${ }^{2}$ \\ ${ }^{1}$ Department of Pediatrics, and \\ ${ }^{2}$ The Edward A. Doisy Department of Biochemistry and Molecular Biology, Saint Louis University School of Medicine, St. Louis, Missouri, USA \\ Address correspondence to: Robert E. Fleming, Department of Pediatrics, Saint Louis University School of Medicine, 1465 South Grand Avenue, \\ St. Louis, Missouri 63104, USA. Phone: (314) 577-8131; Fax: (314) 268-5696; E-mail: flemingr@slu.edu.
}

J. Clin. Invest. 108:521-522 (2001). DOI:10.1172/JCI200113739.

Normal iron homeostasis requires close matching of dietary iron absorption with body iron needs (1). Hereditary hemochromatosis $(\mathrm{HH})$, a common abnormality of iron metabolism, is characterized by excess absorption of dietary iron despite elevated stores, and secondary damage to the liver, pancreas, and other organs (2). Classic HH is caused by mutation of the HFE gene and is inherited as an autosomal recessive trait. However, a substantial percentage of individuals with hemochromatosis, especially in non-Northern European populations, have no mutations in HFE (3). Many such cases differ from classic $\mathrm{HH}$ in the relative distribution of iron between the plasma, hepatocytes, and reticuloendothelial (RE) cells (4).

Pietrangelo et al. recently reported a pedigree with atypical hemochromatosis inherited as an autosomal dominant trait (5). In this issue of the JCI, Montosi et al. report the surprising finding that the gene mutated in these patients (SLC11A3) encodes the iron export protein ferroportin 1 (also known as IREG1, or MTP1) (6). They conclude that the identified mutation (A77D) probably results in loss of ferroportin1 function, suggesting that the affected individuals are haploinsufficient for this gene product. In a nearly simultaneous report, Njajou et al. (7) describe a similar pedigree with autosomal dominant hemochromatosis and a different missense mutation $(\mathrm{N} 144 \mathrm{H})$ in the same gene. Njajou et al., however, conclude that the iron overload phenotype was likely due to gain rather than loss of ferroportin function (i.e., an activating mutation). Why the opposite conclusions?
As shown in Figure 1, ferroportin1 plays key roles in two different aspects of iron homeostasis, absorption of dietary iron by duodenal enterocytes and release of iron from body stores by RE cells (8-10). One might expect a gain-of-function mutation in ferroportin1 to lead to iron loading by increasing iron absorption. Increased ferroportin1 expression in duodenal enterocytes of patients with classic $\mathrm{HH}$ may contribute to their iron loading (11). However, we suggest that the primary cause of iron overload in patients

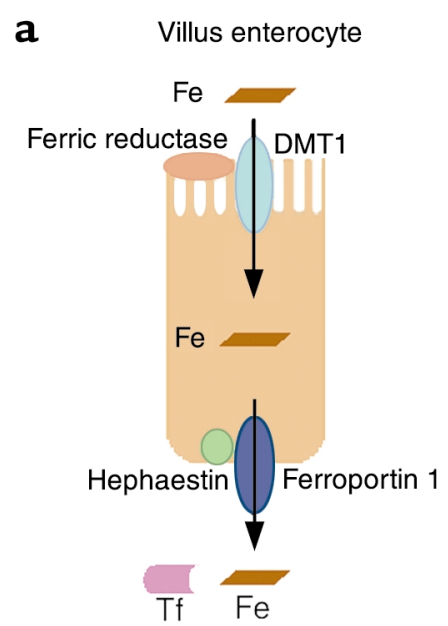

b RE macrophage mutations is decreased ferroportin 1 function in RE cells, rather than increased function in enterocytes. While excess dietary iron absorption in classic HH leads to hepatocellular loading prior to RE cell loading (2), patients with the A77D ferroportin1 mutation demonstrate early and predominant loading of iron in RE cells. This situation is analogous to that seen with defects in the gene encoding ceruloplasmin $(C P)$. The ferroxidase activity of ceruloplasmin converts ferrous iron

Figure 1

Ferroportin 1 in absorption of dietary iron (a) and release of iron stores (b). (a) Dietary nonheme iron at the luminal surface of the villus enterocyte is reduced by ferric reductase and taken up by DMT1. The iron may be retained, or transported across the basolateral surface by ferroportin 1 (blue oval), oxidized by hephaestin, and bound to transferrin (Tf) for plasma transport. (b) Transferrin-bound iron (Tf-Fe) is taken up by the RE cell by a complex containing $\beta_{2}$-microglobulin $\left(\beta_{2} M\right)$, the transferrin receptor (TfR), and the HFE protein (teal oval), which is defective in the classic, autosomal recessive form of hemochromatosis. Heme iron taken up by phagocytosis of senescent erythrocytes (RBC) is released by heme oxygenase (H.O.). The iron may be stored as ferritin, or exported via ferroportin1, after which it is oxidized by ceruloplasmin (Cp), and bound to transferrin for plasma transport. Hephaestin and ceruloplasmin, both indicated by green circles, are structurally similar and perform analogous functions, but their patterns of expression are distinct. Two recent papers, including one in this issue of the $J C l$, have implicated ferroportin in an atypical, autosomal dominant form of hemochromatosis $(6,7)$. 
to the ferric state, which is necessary for iron binding to the plasma transport protein transferrin. Patients with aceruloplasminemia (12) and Cp knockout mice (13) have impaired release of iron from RE cells but unimpaired release of iron from enterocytes, as the enterocyte ferroxidase activity is provided by the ceruloplasmin homologue hephaestin (14). Similarities between patients with aceruloplasminemia and the A77D mutation in ferroportin 1 include increased RE cell iron, subsequent hepatocellular iron loading, relatively low transferrin saturations (despite high ferritin levels), and mild anemia. Patients with the $\mathrm{N} 114 \mathrm{H}$ mutation likewise show hepatocellular iron loading with relatively low transferrin saturations and high ferritin levels. (RE iron status and hemoglobin concentrations of these patients were not discussed.)

Haploinsufficiency for ferroportin would (at least initially) favor low serum iron by decreasing dietary iron absorption and by impairing iron release from macrophages. This could explain the low transferrin saturations, the anemia early in life, and the sensitivity to phlebotomy observed in many of these patients. The hepatocellular iron loading might be explained by the secondary effects of the "erythropoietic regulator" stimulating intestinal iron absorption, or possibly by ferroportin 1 haploinsufficiency in hepatocytes. Ferroportin 1 is also highly expressed in placenta, where it is thought to mediate maternal-fetal iron transport (15). No mention is made in either report of neonatal anemia in these patients, suggesting that haploinsufficiency does not limit this process. However, mutations of both ferroportin 1 alleles in zebrafish lead to early embryonic lethality because of failure of iron transport from the yolk sac (9).

Several questions remain, including whether both the ferroportin missense mutations indeed lead to loss of function, the frequencies of the two mutant alleles, and how haploinsufficiency for ferroportin1 interacts with mutations in HFE. The effect of such interactions is of interest, as it appears that manifestation of the $\mathrm{HH}$ phenotype is greatly influenced by genetic modifiers $(16,17)$. Identifying the specific gene mutations contributing to iron overload should allow genotype-phenotype correlation, leading, in turn, to more accurate genetic counseling regarding prognoses and associated illnesses. Whether a gain- or loss-of-function mutation, each one identified brings us closer to a complete understanding of the many proteins that interact to regulate iron homeostasis.

1. Andrews, N.C. 1999. Disorders of iron metabo lism. N. Engl. J. Med. 341:1986-1995.

2. Bacon, B.R., Powell, L.W., Adams, P.C., Kresina, T.F., and Hoofnagle, J.H. 1999. Molecular medicine and hemochromatosis: at the crossroads. Gastroenterology. 116:193-207.

3. Carella, M., et al. 1997. Mutation analysis of the HLA-H gene in Italian hemochromatosis patients. Am. J. Hum. Genet. 60:828-832.

4. Piperno, A. 1998. Classification and diagnosis of iron overload. Haematologica. 83:447-455.

5. Pietrangelo, A., et al. 1999 . Hereditary hemochromatosis in adults without pathogenic mutations in the hemochromatosis gene. N. Engl. J. Med. 341:725-732.

6. Montosi, G., et al. 2001. Autosomal-dominant hemochromatosis is associated with a mutation in the ferroportin (SLC11A3) gene. J. Clin. Invest. 108:619-623.

7. Njajou, O.T., et al. 2001. A mutation in SLC11A3 is associated with autosomal dominant hemochromatosis. Nat. Genet. 28:213-214.

8. McKie, A.T., et al. 2000. A novel duodenal ironregulated transporter, IREG1, implicated in the basolateral transfer of iron to the circulation. $\mathrm{Mol}$. Cell. 5:299-309.

9. Donovan, A., et al. 2000. Positional cloning of zebrafish ferroportin1 identifies a conserved vertebrate iron exporter. Nature. 403:776-781.

10. Abboud, S., and Haile, D.J. 2000. A novel mam malian iron-regulated protein involved in intracellular iron metabolism. J. Biol. Chem. 275:19906-19912.

11. Zoller, H., et al. 2001. Expression of the duodenal iron transporters divalent-metal transporter 1 and ferroportin 1 in iron deficiency and iron overload. Gastroenterology. 120:1412-1419.

12. Gitlin, J.D. 1998. Aceruloplasminemia. Pediatr. Res. 44:271-276.

13. Harris, Z.L., Durley, A.P., Man, T.K., and Gitlin, J.D. 1999. Targeted gene disruption reveals an essential role for ceruloplasmin in cellular iron efflux. Proc. Natl. Acad. Sci. USA. 96:10812-10817.

14. Vulpe, C.D., et al. 1999. Hephaestin, a ceruloplasmin homologue implicated in intestinal iron transport, is defective in the sla mouse. Nat. Genet. 21:195-199.

15. Gambling, L., et al. 2001. Effect of iron deficiency on placental transfer of iron and expression of iron transport proteins in vivo and in vitro. Biochem. J. 356:883-889.

16. Fleming, R.E., et al. 2001. Mouse strain differences determine severity of iron accumulation in Hfe knockout model of hereditary hemochromatosis. Proc. Natl. Acad. Sci. USA 98:2707-2711.

17. Levy, J.E., Montross, L.K., and Andrews, N.C. 2000. Genes that modify the hemochromatosis phenotype in mice. J. Clin. Invest. 105:1209-1216. 